\title{
XIV. Differential equations of the moon's motion
}

\section{Rev. Brice Bronwin}

To cite this article: Rev. Brice Bronwin (1844) XIV. Differential equations of the moon's motion , Philosophical Magazine Series 3, 24:157, 85-89, DOI: 10.1080/14786444408644808

To link to this article: http://dx.doi.org/10.1080/14786444408644808

$$
\text { 册 Published online: } 30 \text { Apr } 2009 .
$$

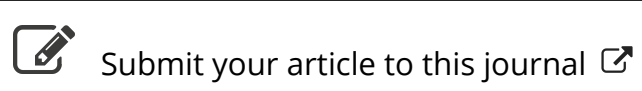

\footnotetext{
Џll Article views: 2
}

Q View related articles $\sqsubset$ 


\section{[85]}

XIV. Differential Equations of the Moon's Motion.

By the Rev. Brice Bronwin*.

IF the moon's coordinates were expressed in terms of the true elliptic longitude instead of the mean, it seems very probable, if not quite certain, that there would be fewer equations. Their development would also be easier. These considerations have led me to seek suitable formula for the purpose, in which I think I have been successful. The object of this paper is to exhibit them.

Let $\mu=m+m^{l}$, the sum of the masses of the moon and sun, $x$ and $y$ the rectangular coordinates of the former on the plane of her orbit, $x^{\prime}, y^{\prime}$ and $z^{\prime}$ those of the latter, $r=\left(x^{2}+y^{2}\right)^{\frac{1}{2}}, r^{\prime}=\left(x^{\prime 2}+y^{\prime 2}+z^{\prime 2}\right)^{\frac{1}{2}}, \quad \mathrm{R}=\frac{m^{\prime}\left(x x^{\prime}+y y^{\prime}\right)}{r^{\prime 3}}$ $-\frac{m^{\prime}}{\left\{r^{\prime 2}-2\left(x x^{\prime}+y y^{\prime}\right)+r^{2}\right\}^{\frac{1}{2}}}$. The equations of motion are

$$
\frac{d^{2} x}{d t^{2}}+\frac{\mu x}{r^{3}}+\frac{d \mathrm{R}}{d x}=0, \frac{d^{2} y}{d t^{2}}+\frac{\mu y}{r^{3}}+\frac{d \mathrm{R}}{d y}=0 .
$$

From these we easily derive

$$
\begin{aligned}
& \frac{d x^{2}+d y^{2}}{d t^{2}}-\frac{2 \mu}{r}+\frac{\mu}{a}+2 \int d \mathrm{R}=0, \\
& d \mathrm{R}=\frac{d \mathrm{R}}{d x} d x+\frac{d \mathrm{R}}{d y} d y=\frac{d \mathrm{R}}{d r} d r+\frac{d \mathrm{R}}{d v} d v,
\end{aligned}
$$

$v$ being the moon's true longitude. Muliplying the first of the above by $x$, the second by $y$, and adding them to the third, we have

$$
\begin{gathered}
\frac{1}{2} \frac{d^{2}\left(r^{2}\right)}{d t^{2}}-\frac{\mu}{r}+\frac{\mu}{a}+\mathbf{P}=0, \\
\mathbf{P}=x \frac{d \mathrm{R}}{d x}+y \frac{d \mathrm{R}}{d y}+2 \int d \mathrm{R}=r \frac{d \mathrm{R}}{d r}+2 \int d \mathrm{R} .
\end{gathered}
$$

Similarly, we find

$$
\begin{gathered}
\frac{x d y-y d x}{d t}-h+\mathrm{Q}=0, \text { or } r \cdot \frac{d v}{d t}-h+\mathrm{Q}=0, \\
\mathrm{Q}=\int\left(x \frac{d \mathrm{R}}{d y}-y \frac{d \mathrm{R}}{d x}\right) d t=\int \frac{d \mathrm{R}}{d v} d t .
\end{gathered}
$$

Now let $g=$ rad, vect. $v=$ long. in the elliptic or undisturbed orbit. These are given by the equations

whence results

$$
\frac{d^{2} \varrho}{d t^{2}}-\frac{h_{0}^{2}}{\rho^{3}}+\frac{\mu}{\rho^{2}}=0, \rho^{2} d \nu=h_{0} d t
$$

* Communicated by the Authol. 


$$
\frac{1}{2} \frac{d^{2}\left(\rho^{2}\right)}{d t^{2}}-\frac{\mu}{\rho}+\frac{\mu}{a_{0}}=0 .
$$

Make $r=\rho+p$, and to abridge $\varepsilon=\mu\left(\frac{1}{a}-\frac{1}{a_{0}}\right)$; for $a$ and $a_{0}, h$ and $h_{0}$ may not be respectively equal, but may differ by quantities of the order of the disturbing force. This value of $r$ being substituted in $\frac{1}{2} \frac{d^{2}\left(r^{2}\right)}{d t^{2}}-\frac{\mu}{r}+\frac{\mu}{a}+\mathbf{P}=0$, making

$$
\frac{d p}{d t}=\frac{d p}{d \nu} \frac{d \nu}{d t}=\frac{h_{0}}{\rho^{2}} \frac{d p}{d \nu}, \quad \frac{d^{2} p}{d t^{2}}=\frac{h_{0}^{2}}{\rho^{4}} \frac{d^{2} p}{d \nu^{2}}-\frac{2 h_{0}}{\rho^{3}} \frac{d \rho}{d t} \frac{d p}{d \nu},
$$

or making $\nu$ the independent variable; in virtue of the assumed equations we find

$$
\begin{gathered}
\frac{d^{2} p}{d \nu^{2}}+p+\frac{\rho^{3}}{h_{0}^{2}}(\mathrm{P}+\varepsilon)+\frac{1}{2 \rho} \frac{d^{2}\left(p^{2}\right)}{d \nu^{2}}+\frac{d\left(\frac{1}{\rho}\right)}{d \nu} \frac{d\left(p^{2}\right)}{d \nu} \\
-\frac{\mu}{h_{0}{ }^{2}} p^{2}+\frac{\mu}{h_{0}^{2} \rho} p^{3}-\frac{\mu}{h_{0}^{2} \rho^{2}} p^{4}+\& \mathrm{c} .=0 .
\end{gathered}
$$

Or if $\frac{1}{\rho}=u$;

$$
\left.\begin{array}{c}
\frac{d^{2} p}{d \nu^{2}}+p+\frac{1}{h_{0}^{2} u^{3}}(\mathrm{P}+\varepsilon)+\frac{1}{u} \frac{d}{d \nu}\left(u^{2} p \frac{d p}{d \nu}\right) \\
-\frac{\mu p^{2}}{h_{0}^{2}} \frac{1}{1+u p}=0
\end{array}\right\} .
$$

The two last terms are of the order of the square of the disturbing force. There will be a term of the form $A \cos (v-\pi)$ arising from the disturbing force, and the quantity $\varepsilon$ or $a$ is to be so determined as to take it away.

The equation $r^{2} d v=d t(h-Q)$ will become by this substitution

$$
d v=\frac{d \nu(h-\mathrm{Q})}{h_{0}(1+u p)^{2}}=d \nu(1+u p)^{-2} \cdot\left(\frac{h}{h_{0}}-\frac{1}{h_{0}^{2}} \int \frac{d \mathbf{R}}{d v} \frac{d \nu}{u^{2}}\right)
$$

From this we shall find $v=b \nu+f(v), b$ being a constant, and $f(v)$ a function periodic of $v$; and we shall have $(b-1) v$ for the progression of the apse. We might have supposed the central force $\frac{\mu}{\rho^{2}}+\frac{\varepsilon}{\rho^{3}}$ instead of $\frac{\mu}{\rho^{2}}$, and have assumed $\frac{d^{2} \rho}{d t^{2}}$ $-\frac{h_{0}^{2}-\varepsilon}{\rho^{3}}+\frac{\mu}{\rho^{2}}=0, \rho^{2} d v=h_{0} d t$, and have found $v=v$ $+f(v)$; but $I$ think the development will be more simple with the assumption above made.

To find the latitude, let $s=\sin$. lat., $i=$ inclination, $\vartheta=$ long. of the node on the plane of the orbit, having a fixed origin on 
that plane. Then $s=\sin i \sin (v-\vartheta)=x \sin v-\lambda \cos v$. We have by known formulæ,

therefore

$$
\frac{d x}{d t}=\frac{\cos ^{2} i}{r^{2} \frac{d v}{d t}} \frac{d \mathrm{R}}{d \lambda}, \frac{d \lambda}{d t}=-\frac{\cos ^{2} i}{r^{2} \frac{d v}{d t}} \frac{d \mathrm{R}}{d x} ;
$$

$$
\frac{d x}{d \nu}=\frac{\rho^{4} \cos ^{2} i}{h_{0}^{2} r^{2} \frac{d v}{d \nu}} \frac{d \mathrm{R}}{d \lambda}, \frac{d \lambda}{d \nu}=-\frac{\rho^{4} \cos ^{2} i}{h_{0}^{2} r^{2} \frac{d v}{d \nu}} \frac{d \mathrm{R}}{d x} .
$$

These formulæ cannot be investigated here, they are found on the assumption that $\frac{d s}{d x} d x+\frac{d s}{d \lambda} d \lambda=0$; consequently $\frac{d s}{d y}$ $=(x \cos v+\lambda \sin v) \frac{d v}{d v}$, and $\frac{d^{2} s}{d v^{2}}+\frac{d v^{2}}{d v^{2}} s=(x \cos v+\lambda \sin v) \frac{d^{2} v}{d v^{2}}$ $+\left(\cos v \frac{d x}{d \nu}+\sin v \frac{d \lambda}{d v}\right) \frac{d v}{d v}$. This becomes, if $\frac{d v^{2}}{d v^{2}}=\mathrm{A}+\Phi$, where $\mathbf{A}$ is constant, $\Phi$ periodic,

$$
\left.\begin{array}{c}
\frac{d^{2} s}{d v^{2}}+A s=(x \cos v+\lambda \sin v) \frac{d^{2} v}{d v^{2}}-s \phi \\
+\frac{\rho^{4} \cos ^{2} i}{h_{0}^{2} r^{2}}\left(\cos v \frac{d \mathrm{R}}{d \lambda}-\sin v \frac{d \mathrm{R}}{d x}\right)
\end{array}\right\} .
$$

All the terms of the second member of this are of the order of the disturbing force.

For correcting the values of the elements $x$ and $\lambda$, we have

$$
x=s \sin v+\frac{\frac{d s}{d v}}{\frac{d v}{d v}} \cos v, \lambda=-s \cos v+\frac{\frac{d s}{d v}}{\frac{d v}{d v}} \sin v .
$$

When we know the parts of $s$ and $v$ depending on the first power of the disturbing force, we can find those of $x$ and $\lambda$ depending on the same by taking the finile variation of the preceding equations; and so on for the higher powers of that force. This method may be employed for correcting the value of $\mathrm{R}$ when we do not wish to find $x$ and $\lambda$ separately. I have employed $x$ and $\lambda$ in preference to the elements $i$ and $\vartheta$, but am not quite sure that it is better to do so.

To find the reduction to the fixed plane, let $\Theta$ be the long. of the node on this plane, $v_{1}$ the long. on it, and make $v-\vartheta$ $=v_{1}-\Theta+\Delta$, or $\Delta=(v-\vartheta)-\left(v_{1}-\Theta\right)$. Then, if $\phi$ $=$ lat. $\sin \Delta=\sin (v-\vartheta) \cos \left(v_{1}-\Theta\right)-\cos (v-\vartheta) \sin \left(v_{1}-\Theta\right)$ $=\tan \frac{i}{2} \tan \phi \cos (v-\vartheta)=\frac{\sin ^{2} \frac{i}{2} \sin 2(v-\vartheta)}{\cos \phi}$, and $\Delta=$ 
$=\sin ^{2} \frac{i}{2} \sin 2(v-\vartheta)\left(1+\frac{1}{2} \sin ^{2} \phi\right)=\sin ^{2} \frac{i}{2}\left(1+\frac{1}{4} \sin ^{2} i\right)$ $\sin 2(v-\vartheta)-\frac{1}{8} \sin ^{2} \frac{i}{2} \sin ^{2} i \sin 4(v-9)=\sin ^{2} \frac{i}{2}\left(1+\sin ^{2} \frac{i}{2}\right)$ $\sin 2(v-9)-\frac{1}{2} \sin ^{4} \frac{i}{2} \sin 4(v-\vartheta)$, neglecting quantities that are insensible. Make $\psi=v+\varepsilon \nu, \theta=\vartheta+\varepsilon \nu, \varepsilon \nu$ being the regression of the node, and

$\mathrm{M}=\sin ^{2} \frac{i}{2}\left(1+\sin ^{2} \frac{i}{2}\right) \sin 2(\psi-\theta)-\frac{1}{2} \sin ^{4} \frac{i}{2} \sin 4(\psi-\theta)$.

Let $\mathbf{M}_{0}$ be the value of $\mathbf{M}$ when $i$ and $\theta$ are changed into $i_{0}$ and $\theta_{0}$, these quantities denoting the constant parts of $i$ and $\theta$. And let $\Delta \mathrm{M}_{0}=\int\left(\frac{d \mathrm{M}}{d i} d i+\frac{d \mathrm{M}}{d \theta} d \theta\right)$, where $\psi$ is regarded as constant both in the differentiation and integration. This will be the part of $M$ depending on the variable parts of $i$ and $\theta$; to which must be added the correction at the origin, or $2 \int \frac{\sin ^{2} \frac{i}{2}}{\cos i} d \vartheta=0$. Then the reduction $=M_{0}+\Delta M_{0}+O$.

We must put for $d i, d \theta$, and $d \vartheta$ their known values. Such equations in $\Delta M_{0}+O$ as have like equations in 0 should be put to the value of $v$; the remainder, if any, with those of $\mathbf{M}_{0}$ nust be separately tabulated.

The coordinates of the disturbing body will be easily expressed in terms of $v$. Thus

$$
n t+\varepsilon=\nu+\mathrm{E}_{1} \sin (\nu-\pi)+\mathrm{E}_{2} \sin 2(\nu-\pi)+.
$$

Or if $\frac{n^{\prime}}{n}=m$,

$$
\begin{aligned}
& n^{\prime} t+m s=m \nu+m \mathrm{E}_{1} \sin (v-\pi)+; \\
& n^{\prime} t+\varepsilon^{\prime}=m \nu+\varpi+m \mathrm{E}_{1} \sin (\nu-\pi)+,
\end{aligned}
$$

where $\varpi=\varepsilon^{\prime}-m \varepsilon$. By this value of $n^{\prime} t+\varepsilon^{\prime}$ we can express $r^{\prime}$, or $u^{\prime}=\frac{1}{r^{\prime}}$, and $v^{\prime}$, the elliptic rad. vect. and long. of the disturbing body in terms of $y$.

We nust not make $m \varepsilon=\varepsilon^{\prime}$, or $\varpi=0$, as has always been done in the lunar theory, unless we afterwards expel $m \nu$ by restoring $\nu^{\prime}$, when $\approx$ will vanish with it.

But I should prefer expressing $t^{\prime}$ and $v^{\prime}$ in terms of $n^{\prime} t+\varepsilon^{\prime}$; or rather I wonld retain $v^{\prime}$, and express $u^{\prime}=\frac{1}{r^{\prime}}$, by means of $r$, and integrate in the manner M. Hansen has done in his Perturbation of Comets. Thus in an abrilged form we have 


$$
\left.\begin{array}{l}
n^{\prime} t+\varepsilon^{\prime}=v^{\prime}+f\left(v^{\prime}\right)=m v+w+m f(v), \\
d v^{\prime}\left\{1+f^{\prime}\left(v^{\prime}\right)\right\}=m d v\left\{1+f^{\prime}(v)\right\}, \\
\text { or } d v^{\prime}=m d v\left\{1+f_{1}\left(v_{1} v^{\prime}\right)\right\}
\end{array}\right\} .
$$

Then integrating by parts,

$$
\begin{gathered}
\int d \nu \sin \left(i \nu+i^{\prime} \nu^{\prime}+\beta\right)=-\frac{1}{i} \cos \left(i \nu+i^{\prime} \nu^{\prime}+\beta\right) \\
-\frac{i^{\prime}}{i} \int d \nu^{\prime} \sin \left(i \nu+i^{\prime} \nu^{\prime}+\beta\right), \\
\int d \nu \cos \left(i \nu+i^{\prime} v^{\prime}+\beta\right)=\frac{1}{i} \sin \left(i \nu+i^{\prime} \nu^{\prime}+\beta\right) \\
-\frac{i^{\prime}}{i} \int d v^{\prime} \cos \left(i \nu+i^{\prime} \nu^{\prime}+\beta\right) .
\end{gathered}
$$

Put for $d v^{\prime}$ its value given by (A.), reduce and integrate again by parts; and so on till the new terms become so small that they may be neglected.

To integrate (1.) make $p=\Sigma \mathrm{A}_{i, i^{\prime}} \cos \left(i \nu+i^{\prime} \nu^{\prime}+\beta\right)$; then

$$
\begin{aligned}
\frac{d^{2} p}{d \nu^{2}} & =-\Sigma A_{i, i^{\prime}}\left(i+i^{\prime} \frac{d \nu^{\prime}}{d \nu}\right)^{2} \cos \left(i \nu+. i^{\prime} v^{\prime}+\beta\right) \\
& -i^{\prime} \Sigma \mathrm{A}_{i, i^{\prime}} \frac{d^{2} v^{\prime}}{d v^{2}} \sin \left(i v+i^{\prime} \nu^{\prime}+\beta\right) .
\end{aligned}
$$

But $v^{\prime} d^{\prime 2} v^{\prime}+2 d r^{\prime} d v^{\prime}=0$, or $u^{\prime} d^{2} v^{\prime}-2 d u v^{\prime} d v^{\prime}=0$, and $d^{2} v^{\prime}=\frac{2 d u^{\prime} d v^{\prime}}{u^{\prime}}=d v^{\prime 2} f_{2}\left(y^{\prime}\right)$. We can therefore eliminate both $d v^{\prime}$ and $d^{2} v^{\prime}$; and after the necessary reductions have been made, it may be convenient to put all the terms after the first with those which arise from the disturbing force. Thus all the integrations required can be performed, and if there be more labour in the integrations, there will be less in the previous developments.

Such is a brief untline of the method 1 would propose for determining the moon's coordinates. It is easier and requires less labour than the old method; cestainly it would require very much less than $M$. Hansen's theory, which is far more laborious than the common method. But general formulæ should be constructed for the determination of the coefficients in the development of the disturbing force after the manner of that author. I may add that it may be applied also to the planets.

Gunthwaite Hall, near Barnsley,

November 25 th, 1843. 